\title{
Primary Gastric ALK-negative EBV-negative Anaplastic Large Cell Lymphoma Presenting with Iron Deficiency Anemia
}

\author{
WEI ZHANG ${ }^{1}$, SAMUEL BURTON ${ }^{1}$, SHAOBIN WU ${ }^{2}$, XIA QIAN ${ }^{2}$, MHD NABEEL RAJEH $^{3}$, \\ KATIE SCHROEDER $^{4}$, MARK SHULDBERG ${ }^{2}$, ADAM MERANDO ${ }^{1}$ and JIN-PING LAI ${ }^{2,5}$ \\ ${ }^{1}$ Department of Internal Medicine, Saint Louis University School of Medicine, St. Louis, MO, U.S.A.; \\ ${ }^{2}$ Department of Pathology, Saint Louis University School of Medicine, St. Louis, MO, U.S.A.; \\ ${ }^{3}$ Division of Hematoloy and Medical Oncology, Saint Louis University School of Medicine, St. Louis, MO, U.S.A.; \\ ${ }^{4}$ Division of Gastroenterology and Hepatology, Saint Louis University School of Medicine, St. Louis, MO, U.S.A.; \\ ${ }^{5}$ Department of Pathology, Immunology and Laboratory Medicine, \\ University of Florida, College of Medicine, Gainesville, FL, U.S.A.
}

\begin{abstract}
Anaplastic large cell lymphoma (ALCL) is a rare subtype of non-Hodgkin lymphoma (NHL). Primary gastric anaplastic lymphoma kinase (ALK) negative ALCL is extremely rare. Diagnosis of primary gastric $A L K$-negative $A L C L$ is difficult to establish and prognosis is worse than ALK-positive ALCL. Here, we report a case of an 82-yearold man with a history of cerebrovascular disease presented with weakness and iron deficiency anemia. He denied any abdominal discomforts. The esophagogastroduodenoscopy revealed a large ulcerated, friable mass in the gastric body which encompassed about $80 \%$ of entire stomach. Biopsy showed a high grade malignant tumor composed of undifferentiated epithelioid atypical cells, making it difficult to determine the cell of origin. Immunostains for lymphoma, carcinoma, and sarcoma were performed. The tumor cells were positive for $C D 30, C D 4$, and $C D 43$, negative for $C D 20$, CD3, ALK-1 and Epstein-Barr virus (EBV)-encoded small RNAs (EBERs) in situ hybridization, establishing the diagnosis of primary gastric ALK-negative ALCL. The patient is currently undergoing chemotherapy with clinical improvement. To the best of our knowledge, this is the first reported case of primary gastric ALK-negative and EBV-
\end{abstract}

This article is freely accessible online.

Correspondence to: Jinping Lai, MD, Ph.D., Department of Pathology, Immunology and Laboratory Medicine, University of Florida, College of Medicine, 1600 SW Archer Road, Gainesville, FL 32610, U.S.A. Tel: +1 3522737842, Fax: +1 3522738172, e-mail: jinpinglai@ufl.edu

Key Words: Stomach, anaplastic large cell lymphoma, ALKnegative, EBV-negative. negative anaplastic large T-cell lymphoma that presented without gastroenterological symptoms.

Anaplastic large cell lymphoma (ALCL), a rare type of NHL, is defined as a malignancy of $\mathrm{T}$ lymphocytes that expresses the lymphocyte activation marker CD30. There are two subtypes of systemic ALCLs: Anaplastic lymphoma kinase (ALK)-positive and ALK-negative ALCL. Patients with ALK-positive ALCL have a better prognosis than those with ALK-negative ALCL. Primary gastric ALK-negative ALCL is extremely rare; and patients usually present abdominal pain $(1,2)$. We report a unique case of ALKnegative ALCL in the stomach who presented with iron deficiency anemia. To the best of our knowledge, this is the first case of primary gastric ALK-negative and Epstein-Barr virus (EBV) negative ALCL that presents without gastrointestinal symptoms.

\section{Case Presentation}

An 82-year-old man with a history of cerebrovascular disease presented with weakness. He denied abdominal discomfort. His physical exam showed a soft abdomen without hepatosplenomegaly. No superficial lymphadenopathy or cutaneous lesions were recognized. Laboratory analysis indicated a white cell count of $9,300 / \mathrm{mm}^{3}$, hemoglobin of $6.8 \mathrm{~g} / \mathrm{dl}$ and MCV of 77.8. His serum iron was $19 \mathrm{mcg} / \mathrm{dl}$, ferritin was $30 \mathrm{mcg} / \mathrm{dl}$ and transferrin saturation was $6 \%$, which were consistent with iron deficiency anemia. He underwent an esophagogastroduodenoscopy which revealed a large ulcerated, friable mass in the gastric body which encompassed about $80 \%$ of entire stomach (Figure 1A). Biopsy showed a high grade malignant tumor composed of undifferentiated cells, making it difficult to determine cell of 
origin (Figures 1B and 1C). Tumor cells contained pleomorphic, hyperchromatic nuclei, and prominent nucleoli with areas of tumor necrosis. They were diffusely arranged in sheets without glandular formations. Immunohistochemical staining was performed with a broad and extensive panel of antibodies trying to characterize the tumor. Markers for lymphoma, carcinoma, and sarcoma were used. CD30, CD4, and CD43 were positive; however, CD20, CD3, ALK and EBV were negative (Figure 2), establishing the diagnosis of primary gastric ALK-negative ALCL. PET scan showed evidence of extensive gastric involvement with lymphoma as well as extensive porta hepatis, peripancreatic, and retroperitoneal lymphadenopathy. A bone marrow biopsy was negative for malignancy. The patient received brentuximab chemotherapy and is currently doing well.

\section{Discussion}

ALCL is a subgroup of NHL first described in 1985 (3). It comprises about $2-3 \%$ of NHL and $12 \%$ of T-cell NHL. ALCL that expresses anaplastic lymphoma kinase (ALK) protein is called ALK-positive ALCL, and has been studied extensively. ALCL lacking ALK has been poorly understood. It was recently accepted as a definite entity in the 2016 World Health Organization (WHO) revised classification system (4). According to recent studies, a unique form of ALK-negative ALCL has arised in association with breast implants and was designated as breast implant - associated ALCL (4).

Patients with ALK-negative ALCL are usually older than those affected by ALK-positive ALCL (5). Although it was proposed that EBV infection may contribute to the pathogenesis of ALCL, recent studies have shown that most cases do not have EBV infection (6). The most common sites of ALK-negative ALCL involvement are lymph nodes. The most frequent extranodal sites of ALK-negative ALCL involvement are skin, liver, and lung, compared to bone and soft tissue in ALK-positive ALCL (7). A few ALK--negative ALCLs involving the stomach have been reported and patients all presented with GI symptoms including epigastric pain, nausea and vomiting. In our case, the patient presented with weakness but without any abdominal symptoms, and was only found to have iron deficiency anemia which prompted an esophagogastroduodenoscopy that identified the gastric mass.

Diagnosis of gastric ALK-negative ALCL is very difficult to establish. Morphologically ALK-negative ALCL is similar to ALK-positive ALCL, although a "small cell variant" is not recognized. Tumor cells are usually large, pleomorphic, with horseshoe-shaped or kidney-shaped nuclei with prominent nucleoli (8). Definitive diagnosis requires further testing by immunohistochemical analysis or flow cytometry. ALKnegative ALCL and ALK-positive ALCL have different immunophenotypes but a positive CD30 stain is required for
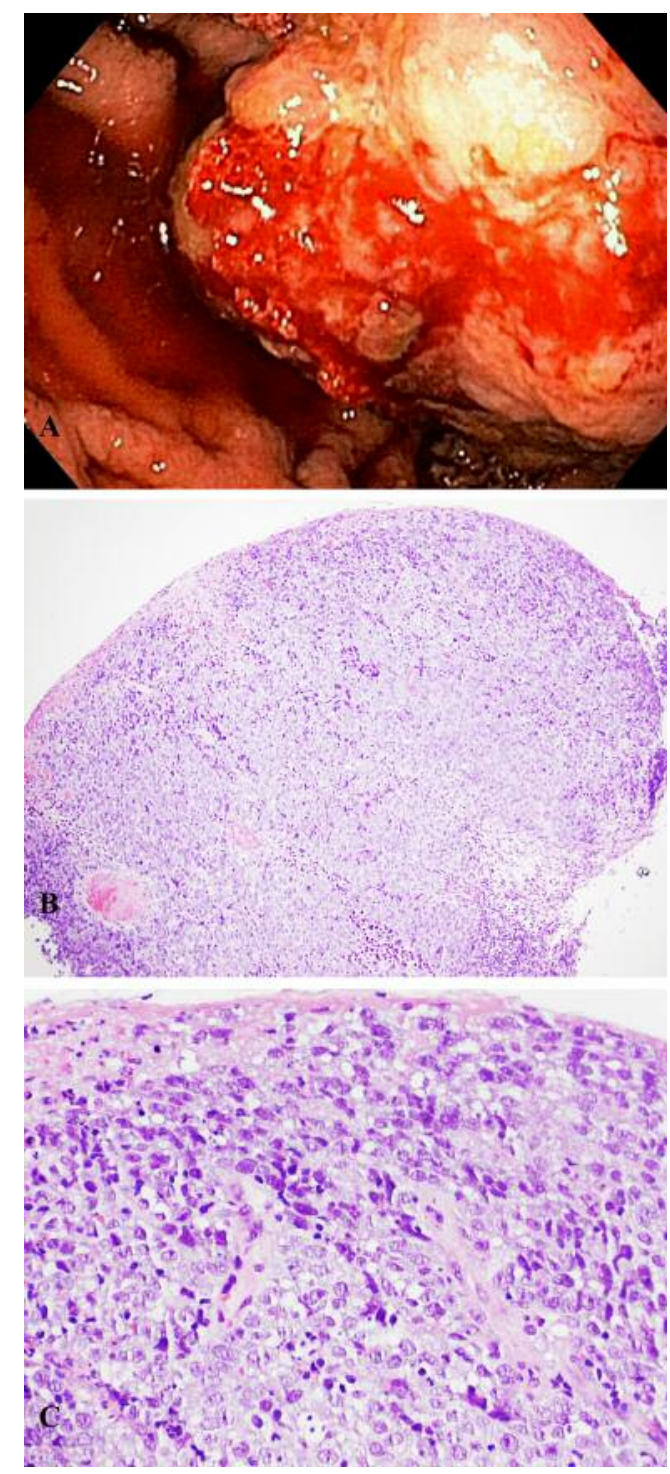

Figure 1. The primary gastric mass. A. Endoscopically, the mass showing ulceration; B-C, Histologically, the tumor is epithelioid and poorly differentiated, with vesicular nuclei and prominent nucleoli (B: $H \& E \times 100, C: H \& E \times 400)$.

the diagnosis of both. In ALK-negative ALCL variable surface receptors including CD3, CD4, CD8, CD43, CD56 and EMA may be expressed (5). The ALK stain distinguishes ALK -negative ALCL from ALK-positive ALCL, due to ALK expression in ALK-positive ALCL but not in ALK-negative ALCL. Our patient's tumor shows diffuse expression of CD30, CD43 and CD4, thus the diagnosis of ALK-negative ALCL was established.

The current mainstay of treatment of ALK-negative ALCL is a regimen of CHOP (cyclophosphamide, doxorubicin, 

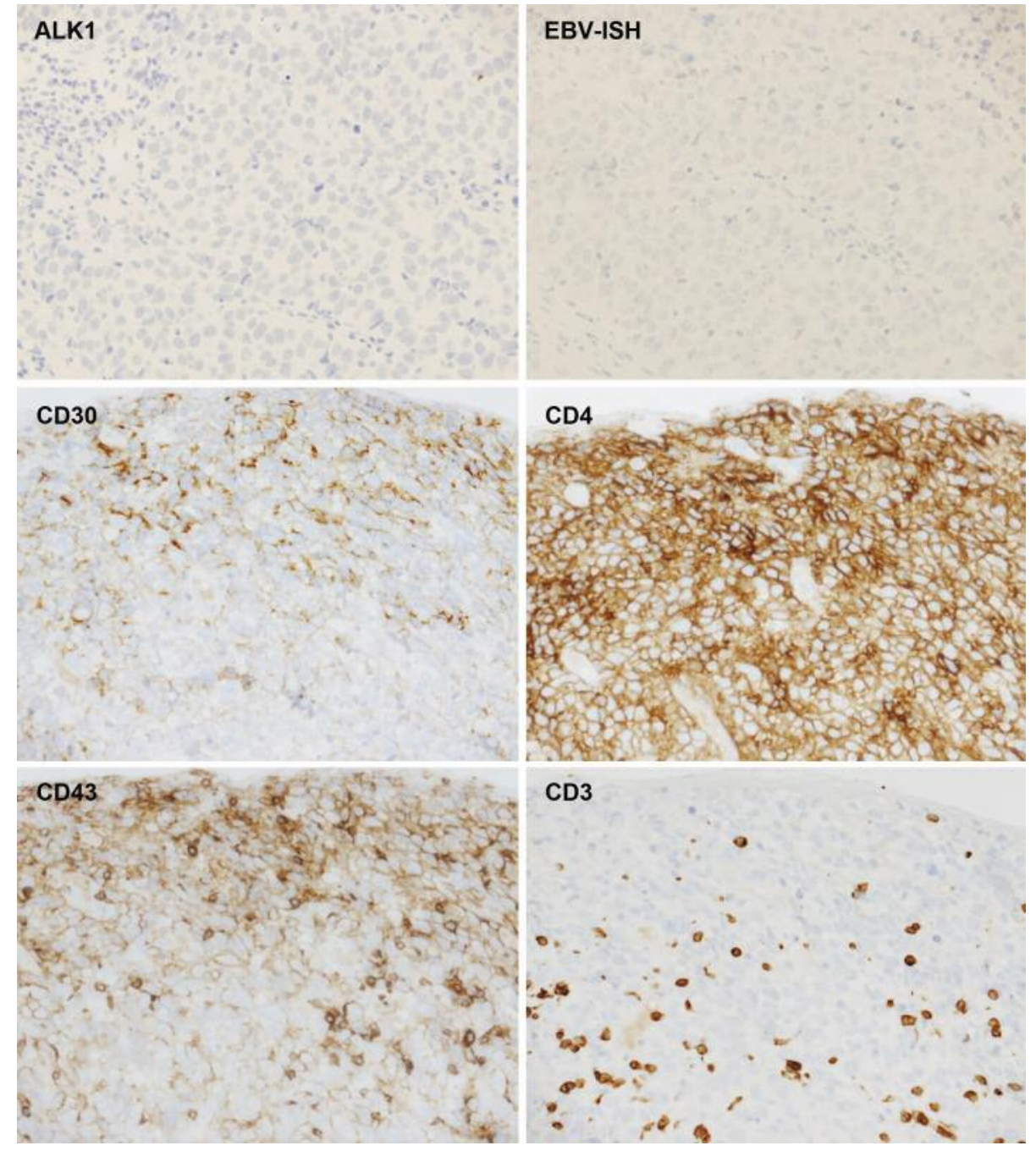

Figure 2. Immunohistochemical analysis and EBV in situ hybridization (ISH) of the gastric tumor. The tumor cells are positive for CD30, CD4, $C D 43$ and negative for ALK1, EBV-ISH, and CD3 ( $\times 400)$.

vincristine, prednisone), followed by autologous stem cell transplantation (ASCT) in first complete remission (9). For relapsed or refractory ALK-negative ALCL, and for patients who cannot tolerate CHOP therapy, brentuximab, an antibody-drug conjugate that targets CD30, can be used. Combination salvage regimens or ALK inhibitors can also be used. Our patient was not a candidate for ASCT, given the advanced age and comorbidities. He received single agent brentuximab and has completed three cycles with clinical improvement.

It was previously reported that ALK-negative ALCL has a poorer prognosis than ALK-positive ALCL. Five-year overall survival was less than $50 \%$ in ALK-negative ALCL and over $70 \%$ in ALK-positive ALCL (5). However, a recent study showed that gene rearrangement may also predict the prognosis of ALK-negative ALCL. It was shown that ALKnegative ALCLs present chromosomal rearrangements of Dual Specificity Phosphatase 22 (DUSP22) or tumor protein p63 (TP63) in 30\% and $8 \%$ of cases, respectively. Five year overall survival in patients with ALK-negative ALCL containing a DUSP22 rearrangement was significantly better than that of patients with a TP63 rearrangement (90\% vs. 17\%) (10). A FISH test for these rearrangements may be indicated and screened routinely in future for patients with ALK-negative ALCL.

In conclusion, we reported a very rare case of primary gastric ALK-negative EBV-negative ALCL in an elderly patient presenting with iron deficiency anemia. Diagnosis of such patients may be difficult and may rely on a biopsy by esophagogastroduodenoscopy if patients had no gastro- 
enterological symptoms. The biopsy revealed the characteristic morphology and diagnosis was confirmed by positive stain of CD30 and variable other T-cell markers and, more importantly, a negative ALK stain. The prognosis of ALK-negative ALCL is generally poorer than ALK-positive ALCL, but may have a more favorable prognosis in patients with DUSP22 rearrangement.

\section{References}

1 Tian $\mathrm{C}$ and Zhang Y: Primary gastric anaplastic lymphoma kinase-negative anaplastic large-cell lymphoma. Onco Targets Ther 9: 5659-5961, 2016.

2 Iwamizu-Watanabe S, Yamashita Y, Yatabe Y, Nakamura S and Mori N: Frequent expression of CD30 antigen in the primary gastric non-B, non-Hodgkin lymphomas. Pathol Int 54: 503-509, 2004.

3 Song HH, Baik GH, Kwon JH, Lee KS, Choi YH, Choi KC and Park YE: A case of primary gastric CD30-positive anaplastic large-cell lymphoma. J Korean Med Sci 20: 1062-1065, 2005.

4 Swerdlow SH, Campo E, Pileri SA, Harris NL, Stein H, Siebert R, Advani R, Ghielmini M, Salles GA, Zelenetz AD and Jaffe ES: The 2016 revision of the World Health Organization classification of lymphoid neoplasms. Blood 127: 2375-2390, 2016.

5 Savage KJ, Harris NL, Vose JM, Ullrich F, Jaffe ES, Connors JM, Rimsza L, Pileri SA, Chhanabhai M, Gascoyne RD, Armitage JO and Weisenburger DD: ALK- anaplastic large-cell lymphoma is clinically and immunophenotypically different from both ALK+ ALCL and peripheral T-cell lymphoma, not otherwise specified: report from the International Peripheral TCell Lymphoma Project. Blood 111: 5496-5504, 2008.
6 Herling M, Rassidakis GZ, Jones D, Schmitt-Graeff A, Sarris $\mathrm{AH}$ and Medeiros LJ: Absence of Epstein-Barr virus in anaplastic large cell lymphoma: a study of 64 cases classified according to World Health Organization criteria. Hum Pathol 35: 455-459, 2004.

7 Ferreri AJ, Govi S, Pileri SA and Savage KJ: Anaplastic large cell lymphoma, ALK-negative. Crit Rev Oncol Hematol 85: 206215, 2013.

8 Benharroch D, Meguerian-Bedoyan Z, Lamant L, Amin C, Brugieres L, Terrier-Lacombe MJ, Haralambieva E, Pulford K, Pileri S, Morris SW, Mason DY and Delsol G: ALK-positive lymphoma: a single disease with a broad spectrum of morphology. Blood 91: 2076-2084, 1998.

9 Hapgood G and Savage KJ: The biology and management of systemic anaplastic large cell lymphoma. Blood 126: 17-25, 2015.

10 Parrilla Castellar ER, Jaffe ES, Said JW, Swerdlow SH, Ketterling RP, Knudson RA, Sidhu JS, Hsi ED, Karikehalli S, Jiang L, Vasmatzis G, Gibson SE, Ondrejka S, Nicolae A, Grogg KL, Allmer C, Ristow KM, Wilson WH, Macon WR, Law ME, Cerhan JR, Habermann TM, Ansell SM, Dogan A, Maurer MJ and Feldman AL: ALK-negative anaplastic large cell lymphoma is a genetically heterogeneous disease with widely disparate clinical outcomes. Blood 124: 1473-1480, 2014.

Received March 16, 2017

Revised April 9, 2017

Accepted April 10, 2017 